\title{
L'HOMME L'Homme
}

$202 \mid 2012$

Varia

\section{La possession par l'écriture}

\section{André Mary}

\section{OpenEdition}

\section{Journals}

Édition électronique

URL : http://journals.openedition.org/lhomme/23069

DOI : 10.4000//homme.23069

ISSN : 1953-8103

\section{Éditeur}

Éditions de l'EHESS

\section{Édition imprimée}

Date de publication : 4 juin 2012

Pagination : 193-204

ISSN : 0439-4216

\section{Référence électronique}

André Mary, «La possession par l'écriture », L'Homme [En ligne], 202 | 2012, mis en ligne le 29 mai 2014, consulté le 03 mai 2019. URL : http://journals.openedition.org//homme/23069 ; DOI : 10.4000/ Ihomme.23069

(c) École des hautes études en sciences sociales 


\title{
La possession par l'écriture
}

\author{
André Mary
}

O

N PEUT AVOIR SUIVI de longue date le parcours de vie et l'entreprise d'écriture de Marc Augé, l'avoir souvent rencontré et écouté, avoir tout lu de ses publications savantes et de ses "petits livres ", reconnaître les lieux et les personnages qui sont évoqués ici, cette "vie en double " est une réelle découverte et si l'on peut dire "un vrai livre ", plus que tout autre le témoignage d'un re-commencement toujours possible, une promesse de vie.

\section{Du mélange des genres}

L'auteur, allergique à l'égotisme littéraire, précise d'emblée que « ce livre n'est pas une autobiographie» comportant quelque révélation sur sa vie privée et intime mais il s'agit bien, au-delà de quelques détails biographiques, de l'autoportrait d'une vocation, de ce qui fait sens dans une vie personnelle et professionnelle. Parler de soi ou écrire sur soi est devenu aujourd'hui un genre - et même un impératif - si répandu et en même temps tellement problématique qu'il y a de quoi s'y perdre. Pour s'en tenir à l'espace d'écriture des anthropologues, il est clair que ce parcours de vie avec ces images souvenirs, ces moments d'émotions et de rencontres de personnages et d'amis, ne relève pas de l'exercice d'analyse rétrospective d'un itinéraire intellectuel ou de la construction du parcours «logique » d'une ouvre, genre auquel l'auteur s'est déjà livré ${ }^{1}$. Plus surprenant, il ne s'agit pas non plus d'une œuvre inspirée d'un journal de terrain ou d'enquête.

1. Cf. Marc Augé, «Qui est l'autre? Un itinéraire anthropologique », L'Homme, 1987, 103 : 7-26.

À propos de Marc Augé, La Vie en double. Ethnologie, voyage, écriture, Paris, Payot \& Rivages, 2011 ( Manuels Payot»). 
Sans nier l'intérêt du journal de bord pour soi comme pour les autres, en tant qu'archives de la recherche, l'ethnologue de métier déclare n'avoir jamais vraiment tenu de journal de terrain (p. 210). Sur le fond, la bibliographie de Marc Augé renvoyant à ses périodes de terrains " classiques " ne relève pas du paradigme de l'écriture «en double» que Vincent Debaene s'attache à repérer dans la production des ethnologues français : d'un côté la monographie savante, de l'autre le journal ou l'écriture littéraire ${ }^{2}$. Même si toute monographie a "sa part de littérature ", Le Rivage alladian (1969), Théorie des pouvoirs et idéologie (1975), ou Le Dieu objet (1988), dont les "terrains " sont évoqués ici par images et témoignages ponctuels, n'ont pas de double littéraire. Aujourd'hui encore, bien que, comme l'aurait dit Leiris, tout ethnologue soit hanté " par le fantôme de l'autre livre» (cité p. 232), point de rêve ou de regret avoué chez Marc Augé de ne pas avoir écrit l'équivalent de Tristes Tropiques ou de L'Afrique fantôme, ou même de L'Afrique ambiguë.

Il ne s'agit pas d'une position de principe sur le mélange des genres et encore moins d'une suspicion par rapport à l'écriture littéraire, puisque, au contraire, à son retour dans l'hexagone et notamment avec cet « ethnoroman » de La Traversée du Luxembourg (écrit en 1985), Marc Augé inaugure un genre fondé sur le mélange ou l'assemblage de fragments de vie, de fenêtres ethnologiques, de moments d'ethnofiction, d'images d'ici et d'ailleurs ou de mini-leçons d'anthropologie, avec relecture des grands auteurs, sur ses thèmes favoris: le rituel, le moi et l'autre, la mémoire et l'oubli. Mélanges ou alternance, on peut parler d'une implosion du genre "ethnologique " cherchant à explorer quasi expérimentalement toutes les virtualités d'expression inexploitées jusqu'alors.

$\mathrm{La}$ "vie en double» marque cependant une pause par rapport aux exercices d'ethnologie "inversée ", au journal d'actualité et aux carnets de route, à la "littérature expérimentale» de l'ethnoroman ou de l'ethnofiction, ou au genre de l'essai par "petits textes ", même si le chapitre de cet ouvrage sur "Anthropologie et littérature", et le retour sur "Lieux et non-lieux» constituent des contributions décisives sur ces sujets. L'originalité de ce "dernier» ouvrage s'inscrit dans une temporalité plus délibérément autobiographique qui fait place à des facettes inédites (souslieutenant Augé de l'armée française, mon «ami Gbagbo ", entrouvre la porte de lieux centraux (de la «station » ORSTOM à l' "École» et ses « séminaires »), et témoigne de rencontres marquantes (les architectes, les Grands hommes). Le fondu enchaîné de l'évocation des terrains, des événements,

2. Cf. Vincent Debaene, L'Adieu au Voyage. L'ethnologie française entre science et littérature, Paris, Gallimard, 2010. 
des rencontres et des moments de réflexion, est particulièrement soigné et réussi : on mesure le chemin parcouru et le travail sur soi accompli. L'impression de familiarité avec les personnages rencontrés (prophètes, chamanes ou hommes d'État) et les paysages traversés (la baie d'Along ou l'espace des Llanos de l'Apuré) laissent place à l'irruption d'images fortes d'émotion ou à des souvenirs complices (on pense au plaisir de la conduite sur piste et au "moment parfait " de survol de la tôle ondulée), autant de bonheurs d'écriture. Nul doute que Marc Augé est un écrivain mais aussi un "auteur" qui a son style d'écriture, ses mots et ses jeux de mots, ses formules bien frappées: "les vrais lieux sont en nous ". La revisite de ses thèmes favoris est l'occasion de trouvailles toujours sensibles à l'ambiguïté du réel et à l'ironie des situations: l'inversion comme "vaccination symbolique»; le prophète "annonciateur» ou "provocateur»; «le nonlieu par exténuation ".

Les lunettes freudiennes ou bourdieusiennes de l'auto-analyse du passé sont ignorées par ce travail d'écriture continuée et régulièrement réamorcée qui flirte avec les vertus proustiennes du temps retrouvé (p. 220). Bien sûr, comme toute écriture relevant de la "confession" et du retour sur soi, il s'agit bien d'un rituel mais l'esprit de l'aventure se veut étranger au ressassement du passé, à l'examen de conscience ou aux Mémoires. Le message est celui d'une promesse de vie, d'un appel d'expériences et d'un commencement ressuscité, tourné vers l'avenir, le sens du projet, une préoccupation récurrente des derniers écrits qui aimerait se dire, comme le dernier mot de ce livre "qui pourrait être le dernier", que «la mort n'existe pas». On pourrait parler en termes quasi augustiniens (sans faire offense à un païen convaincu), d'une reconfiguration "poïétique " de soi par l'écriture - l'aveu des péchés en moins, ce qui n'est pas rien. L'auteur parle d'" une tentative de généalogie de [sa] relation à l'écriture qui invite d'autres à l'exercice d'épure » (p. 106).

\section{Terrains initiatiques}

Si le mot premier et dernier de ce livre est l' "écriture " (le géométral de toutes les perspectives ?), l'ethnologue rappelle avec fermeté que le "terrain " comme expérience, n’en déplaise à ceux qui réduisent l'ethnologie à la production ou l'analyse de textes, est ce qui donne sens à son métier : "Sans son incessante confrontation avec ses "terrains", l'anthropologue ne pourrait plus écrire: ils font son inspiration et lui donnent forme» (p. 221).

Sur cette expérience "initiatique " du terrain, Marc Augé ne varie pas et $\mathrm{y}$ revient, même si la palette des situations que recouvre le terme de 
«terrains " prend ici toute son extension et intègre de multiples déclinaisons. Des ethnologues de renom ont alimenté à leur manière la métaphore militaire du terrain : on pense à Evans-Pritchard bien "campé " par Clifford Geertz. Le service militaire en pleine guerre d'Algérie se présente de fait comme une initiation au "terrain", non seulement par quelque concession au langage des instructeurs, mais parce qu'il est vécu comme une confrontation à ce que Bourdieu appelle la "violence du symbolique", ce que le jeune normalien agrégé Marc Augé traduit en termes d'efficacité de l'« a priori du symbolique » : la logique complexe des relations prescrites qui régissent un monde de "gradés " et les ruses symboliques qu'elles comportent (se retrouver à "faire la chèvre " dans un "piège à fellaghas " p. 39). Le transfert d'analogie aux relations "prescrites" qu'impose le terrain classique de l'ethnologue et notamment aux rapports à la fois amicaux et paternalistes du chercheur à son informateur ne manque pas d'audace mais, comme le confirmeront les relations de préséance qui marquent les premiers contacts avec la Côte-d'Ivoire, en ville comme au village, de Jacqueville à Yamoussoukro, le terrain est politique de part en part et n'échappe pas à la "situation coloniale». Marc Augé n’a pas choisi ou eu l'opportunité d'investir ce premier "terrain» algérien en le prolongeant, à la manière de Bourdieu, par une ethnologie des sociétés locales et des cultures "indigènes ": la description "dense " de la plage d'Oran comme introduction à la situation globale de l'Algérie de l'époque restera une virtualité inaccomplie.

Le vrai terrain initiatique sera celui du rivage alladian, ainsi en ont décidé les "dieux ", et plus particulièrement Georges Balandier. Mais ce déplacement "sédentaire " inaugural, à bonne distance d'Abidjan, en introduit bien d'autres: d'abord celui qu'impose le suivi même des groupes sociaux et des sujets étudiés vers le village thérapeutique de Bregbo, ce premier hors lieu ou "non-lieu ", puis l'approche comparative qu'apporte le terrain des cultes de possession togolais. Marc Augé ne s'attarde pas sur les pratiques du "double terrain » ou de l'ethnographie «multi-sites " et autres formules à la mode, sans doute parce que, entre le terrain premier de longue durée qu'il a connu et qui l'a formé, et l'ethnologie de parcours ou de séjours, de visites et revisites de courtes durées, isolées ou accompagnées, ou cette ethnologie voyageuse et "itinérante" (p. 262) qui pratique des chemins de traverse, l'écart est à la fois indépassable et irremplaçable. En même temps, cette expérience inaugurale initie une posture de "déracinement chronique", un besoin de sortie de soi et de dépaysement, toujours associés au double sédentaire qui reste ou qui pousse à revenir, et que l'on retrouve au cœur de l'itinérance des voyages du Président de l'École des hautes études en sciences sociales. 
Le terme que retient l'anthropologue pour ces autres terrains « d'incursions " qui ont été, pour les plus marquants, les terrains des autres (Brésil, Venezuela) est aujourd'hui celui de "rencontre». Toute expérience ethnographique, dira-t-on, est une rencontre avec l'autre "indigène " en même temps qu'une confrontation à soi comme autre, mais toutes les situations d'observation quotidienne des autres et de soi comme autre, ou les occasions de rencontres fortuites offertes par l'itinérance, ne sont pas des "rencontres» au sens fort, disons anthropologique, du terme. Une rencontre suppose un déplacement de soi vers l'autre, mais plus important encore et plus rare, la réciprocité d'un déplacement de l'autre par rapport à lui-même qui permet un croisement de perspectives. Ce qui rend possible la rencontre "anthropologique" idéale, c'est une expérience partagée, "en double ", de l'altérité qui prend du temps et que Marc Augé nous dit avoir vécu au plus fort avec son "compagnon » et ami Boniface (p. 139), et qu'il imagine avoir été celle de Bernard Maupoil et de son informateur avisé Gèdègbe. Il est significatif que cette situation relationnelle et épistémique de rencontre ne soit possible que dans un "espace intermédiaire " et par la médiation d'un tiers, l'informateur et interlocuteur devenu avec le temps un compagnon, ou par ces médiateurs et amis que sont les collègues, doctorants, qui ont fait un bon bout du chemin chez les autres. On pourrait parler, si le terme n'évoquait pas aujourd'hui autre chose et notamment la co-écriture (une virtualité que l'ethnologue écrivain n'aborde pas), d'ethnologie "collaborative», ou plus simplement d'une ethnologie associative ou de " compagnie " dont le principe minimal serait un principe de triangulation: l'ethnologue, l'autre et le tiers médiateur, l'informateur "privilégié ", l'indigène "éclairé " ou l'ethnologue adopté. On sait à quel point, dans ces "incursions" chez les autres, en équipe, le dispositif filmique a joué un rôle non négligeable de révélateur ou d'embrayeur de communication, facilitant le déplacement des autres par rapport à l'image de soi (et réciproquement).

On ne peut que s'interroger, en revanche, sur cette anthropologie du monde global où l'ethnologue serait «à lui-même son propre informateur, ou en tout cas l'un de ses informateurs privilégiés » (p. 260), la seule médiation étant ici le dispositif de l'écriture (et le lecteur?). Peut-on néanmoins encore parler de "rencontre", au sens dialogique du terme, dans une ethnologie de l'itinérance ou de l'occasion? Dans Le Métro revisité (p. 26) ${ }^{3}$, l'ethnologue des autres voyageurs reconnaît que sa «matière ethnologique " s'apparente plutôt dans ce cas de figure à la "matière du 
romancier » qui s'efforce de "pénétrer la subjectivité d'autrui » par l'autoanalyse ou l'autofiction. Mais l'entreprise atteint ses limites au regard de l'altérité revendiquée des "jeunes" des banlieues: il faudrait en " rencontrer» au moins un et pouvoir lui parler pour briser son opacité.

\section{Je est un autre: l'écriture ou la possession "idéale"}

La question du moi et de l'autre est au cour de «l'anthropologie de l'anthropologie " de Marc Augé et la figure du double en est, si l'on peut dire, le noyau puisqu'être soi n'est jamais simple. Cette vie "en double» prend souvent les allures mondaines d'une vie multiple: l'ethnologue romancier, le président écrivain, le voyageur et globe-trotter, mais aussi le Professeur ou le Directeur d'études. Ce qui importe, c'est que chaque figure de soi ou chaque place, est travaillée par son double, le paradigme du sorcier, "celui qui sort de soi ", à l'image de l'ethnologue, constituant dans ce parcours anthropologique l'initiation première à cette relation ambiguë et ambivalente du soi et de l'autre que l'on retrouve chez l'écrivain. Avec le temps, en passant notamment par la culture vodou du Togo et les transes chamaniques de l'Amérique du Sud, la "matière ethnologique » de la possession (et du rêve) a pris une place majeure et omniprésente, constamment revisitée, dans cette anthropologie du double. Cette expérience de dédoublement avec perte de soi ou oubli de soi (dont l'auteur ne fait jamais état de quelque vécu personnel sauf par le biais des rencontres du monde chamanique du rêve par lesquelles il découvre avec émotion qu'on peut " exister" pour les autres, ailleurs) a toujours exercé sur lui une fascination incontestable. Ses usages métaphoriques donnent à penser les zones liminales de l'existence humaine et suppléent à leurs impasses narratives, qu'il s'agisse de la "mort d'avant la naissance ", cet état premier d'indistinction du moi et de l'autre, ce "cauchemar mythique" pour reprendre les termes de Walter Benjamin (cité p. 223), ou des images et des rêves qui ne cessent de vous hanter et vous poursuivre, comme en témoignent les accents " animistes » de la fin de ce livre, en espérant trouver la survie sinon dans un autel du moins dans un autre livre à venir.

Pour éviter que la vie en double ne devienne une vie "en diable" n'ayant d'autre issue que la mort dévorante ou la folie du délire, il existe heureusement les ressources de médiation du tiers: le prophète, le chamane, la mère de saint, et les dispositifs rituels de la vision ou de la possession. On comprend que l'écrivain voyageur soit aujourd'hui sensible aux "impasses narratives " que représentent ces mises en récit sous contrôle rituel et leurs logiques des signes inscrites dans le monde de l'oralité et 
du mythe. L'écriture est plus que jamais pour lui comme pour bien d'autres (même si elle reste un rituel) une ressource décisive notamment au plan de la sortie du "cauchemar mythique " et de la construction de soi, et la littérature l'instrument d'une libération. L'expérience de l'écriture comme dédoublement, le je est un autre, c'est au fond la "possession idéale»: "celle où l'on n'est possédé que par soi-même, non pas dépossédé de soi, mais en reconquête de son passé le plus lointain " (p. 118), celle qui évite aussi de se prendre pour un dieu (sauf pour certains). C'est aussi par l'écriture que l'ethnologue du "lieu anthropologique » et le voyageur des nonlieux pris dans le tourbillon du "nomadisme planétaire " se retrouvent dans une même posture proprement anthropologique de croisement des perspectives (p. 256).

\section{Anthropologie et littérature}

Si l'anthropologue a besoin de l'écriture et si l'écriture a une vocation pré-anthropologique par la distance à soi et l'intégration du point de vue de l'autre qu'elle implique, toute écriture n'est pas pour autant anthropologique et toute production de texte n'a pas valeur ethnographique, sauf à jouer sur ce double sens de l'anthropologie qui travaille toute l'œuvre de Marc Augé et que condense la formule répétée : "l'anthropologie est toujours une anthropologie de l'anthropologie des autres". Le chapitre «Anthropologie et littérature » est sur ce point un "petit essai » qui est sans nul doute le cœur de l'ouvrage. Les références littéraires (de Proust à Chateaubriand) sont comme toujours omniprésentes dans le texte de Marc Augé mais il n'est pas question ici d'une anthropologie de la littérature ou des écrivains envisagée comme nouveau champ de l'anthropologie, ni encore moins des rapports entre l'ethnologue de la littérature orale et le " critique littéraire » évoqués en passant, mais d'interroger les relations entre anthropologie et littérature dans leur recouvrement et leurs différences.

Pour simplifier, on peut dire que l'auteur soutient une thèse à double facette, l'une sur la forme, l'autre sur le fond, qui semble entretenir l'idée d'un certain continuum entre anthropologie et littérature, même si la complexité de cette "matière formelle" commune (p. 222) exige plus d'attention, et que le sens des écarts sur les référents et les finalités ressurgit in fine. Sur la forme, tout individu qui, dans une société donnée et à son époque, observe (les autres ou soi comme autre, on n'y reviendra pas), qui prend note dans quelque carnet et qui écrit pour être lu par quelque autre, ses partenaires institutionnels ou ses collègues spécialisés, peut être considéré comme un écrivain. Tout écrivain n’est pas pour autant un "auteur" témoignant d'un style au sein d'un genre donné, mais l'écart 
des genres et encore moins des styles d'écriture ou de rhétorique ne permet pas de tracer une frontière nette entre l'œuvre littéraire, le roman et la monographie ethnologique. Entre le descriptif littéraire et le documentaire démonstratif, les recouvrements sont nombreux: toute monographie, comme on l'a dit, a "sa part de littérature ", et les grandes œuvres littéraires, on le sait, ont leur part d'ethnographie (Proust étant décidément l'exemple même du "naturel » ethnologique, au sens premier il est vrai, p. 219). La multiplication du genre ethnologique recourant aux histoires de vie, à l'autobiographie et même à la fiction (Marc Augé se retrouvant sur ce sujet en phase avec Michael Taussig pour comprendre que la fiction est parfois plus "vraie" que la description, p. 217) ne fait que confirmer cette production d'un continuum ethnolittéraire. Notons que Clifford Geertz a contribué à faire reconnaître cette osmose des figures du discours anthropologique en adoptant, notamment dans son fameux essai sur la thick description, un texte ethnographique qui ne porte pas sur un objet ou un rite comme le fameux combat de coq à Bali, mais sur un récit narratif ${ }^{4}$.

Sur le fond, le référent du discours, la thèse de la continuité peut sembler aussi prévaloir : la "matière première " des expériences humaines et des observations dont se nourrissent l'ethnologue et le romancier n'estelle pas la même? Les questions et réflexions sur la vie et la mort, le moi et l'autre, de l'anthropologue sont, après tout, au-delà des manières de dire et des degrés de réflexivité, issues du " simple constat d'une vérité humaine que chacun peut entendre» (p. 219). On mesure le glissement possible vers le socle d'un questionnement méta-anthropologique du type "qui sommes-nous? où allons-nous?", qui ferait de tout homme un métaphysicien à ses heures autant qu'un anthropologue, non sans lucidité et réflexivité sur sa condition, ne croyant pas vraiment à ses mythes et ses rites? Mais on ne rencontre pas un informateur avisé et éclairé comme Gèdègbe ou un romancier "naturellement » ethnologue tous les jours, et il est difficile de faire de l'expérience anthropologique une expérience humaine "commune". Marc Augé le sait mieux que d'autres et nous rappelle que les structures pré-narratives de l'expérience humaine qu'évoque un phénoménologue comme Paul Ricœur (cité p. 226) sont bien le produit, y compris dans les sociétés de l'oralité, de mises en forme symbolique, historiquement et culturellement situées, des cosmologies et des anthropologies locales. Les questions ultimes sur les vivants et les morts n'ont pas de sens commun en dehors de leur formulation, en un mot "toute matière est formelle" et peut difficilement, à ce titre, être commune. L'ethnologue vient surtout nous rappeler qu'à l'inverse toute

4. Cf. Clifford Geertz, The Interpretation of Cultures, New York, Basic Books, 1973. 
expérience humaine n'est pas "anthropologique». L'interrogation sur "ce en quoi nous vivons » qui est au cœur de l'expérience ethnographique, les situations vécues d'incursion, d'interlocution et de "rencontre " au sens anthropologique du terme, n'est pas a priori celle que tout homme peut entendre.

L'ambiguïté pourrait être levée mais sans doute ne ferait-elle que se déplacer, si le double sens de l'anthropologie ne travaillait de l'intérieur cette confrontation globale entre anthropologie et littérature. Les anthropologies "locales" - spontanément assimilées aux anthropologies " païennes " - sont-elles encore à penser en termes de "théories indigènes " à la manière de Lévi-Strauss qui les distinguait non seulement des ethnologies singulières mais de l'anthropologie au sens comparatif du terme? Marc Augé ne remet nullement en cause le credo anthropologique qui prétend transcender la simple restitution de l'expérience singulière d'une société et rechercher le géométral de toutes les perspectives locales, non pas tant à partir de la position de survol de l'astronome qu'à partir de l'exemplarité et de l'universel concret des études de cas (p. 230). C'est donc dans un espace encore une fois triangulaire, non spéculaire, entre une ethnologie anthropologique, les littératures "régionales» inscrites dans des mondes et des lieux, et les anthropologies locales, que la question mérite d'être prolongée.

\section{"Les vrais lieux sont en nous"}

De Jacqueville à Combray, du suivi " partagé » des confessions du village thérapeutique d'Atcho à la posture d'observation intime des conversations de salon des Guermantes, la notion de «lieu anthropologique» fait son chemin et le chapitre sur lieux et non-lieux revient utilement sur les malentendus et les perplexités suscités par l'anthropologie "négative" $\mathrm{du}$ « non-lieu » en concluant que : «Les vrais lieux sont en nous». S’il est un lieu qui habite et qui hante le "Président écrivain" - surtout quand il est ailleurs - et auquel « il doit tout» (p. 105), c'est bien « l'École», pour reprendre le langage mythique des élus; non pas - pour les non-initiés l'École normale ou l'École pratique (EPHE), mais l'École des hautes études en sciences sociales, un lieu un et multiple. Le contexte imposait un chapitre surtout quand le «lieu historique et symbolique » de l'institution et sa direction, à laquelle Marc Augé a toujours été associé dès la création de l'EHESS, se trouve confronté à une politique de campus vécu comme un hors lieu. Comme l'observe l'anthropologue de la globalisation: «La logique du lieu et du micro-contexte est en voie de perdre sa place dans un monde marqué par l'immersion du lieu dans le non-lieu» (p. 251). 
S'il est vrai que les institutions sont mortelles, c'est sans doute le moment historique pour esquisser une histoire et accumuler les matériaux ethnobiographiques d'une ethnologie rétrospective d'un lieu menacé d'exténuation? Marc Augé nous livre ici et ailleurs par fragments et portraits, comme pour le métro, les pistes d'une lecture de ce lieu rituel et de ces rites d'institution: la voie royale du normalien agrégé; le séminaire, lieu de fidélités et de rencontres, de "confessions publiques " et d'improvisations théâtrales; et puis le grand jeu des élections et des filiations, tout un monde construit sur des règles de bonne distance et de préséance, sur fond d'amitiés entre camarades de promotion et d'admiration pour les Grands Ancêtres. On ne sera pas surpris de retrouver l'engagement du Président de ce Grand Établissement pour les valeurs de liberté, de diversité et d'excellence personnelle, hostile au nouvel esprit de la politique de la recherche, avec ses directions de programme et même ses directions de thèse (une bonne thèse, comme le disait un directeur d'études, est d'abord une thèse qui n'a pas besoin d'être dirigée...). La philosophie profondément libérale qui s'affirme n'est pas sans lien avec le constat des impasses collectives de la génération "critique » des Héritiers et nous éloigne des élans et des amitiés socialistes d'antan. La conclusion de Carnets de route et de déroutes est très claire : « La gestion raisonnable des affaires publiques ne devrait-elle pas avoir pour but et pour idéal, lui-même utopique, de laisser chacun vaquer aussi librement que possible à ses "occupations" ?" 5

\section{Prophètes des temps derniers}

Le «déplacement chronique» de l'anthropologue pour les terres lointaines a traditionnellement pour envers le péché d'allotopie, le renvoi de l'autre dans une autre temporalité. L'anthropologue des «mondes contemporains" n'ignore pas pour autant les limites de la posture de contemporanéité, notamment au regard des défis inhérents au décalage de génération. Il fait régulièrement de sa non-contemporanéité par rapport à des mondes comme celui des "jeunes", pas seulement des banlieues, un objet de méditation, en soulignant qu'elle n'est pas en soi insurmontable. On peut s'interroger par contre, en faisant retour sur le terrain de ses rencontres initiatiques premières, sur cette mort annoncée et répétée des prophètes qui prend la forme de l'aveu d'une perte de contemporanéité par rapport au phénomène prophétique ivoirien 
actuel : «Les derniers prophètes sont morts aujourd'hui » (p. 196). On aura compris que Marc Augé parle ici des prophètes paysans guérisseurs qu'il a connus - qualifiés pourtant par leurs ruses symboliques d'"annonciateurs " ou de "provocateurs»-, mais désormais voués à disparaître de la scène, leur mort et la fin d'un monde accompagnant de façon significative le départ et le retrait de l'ethnologue qui tire le rideau. Leur " fétichisation " anthropologique comme objets de mémoire, soubresauts des impasses de la matrice païenne, fait paradoxalement écho au discours des nouveaux pasteurs et prophètes des mouvements qualifiés de "protestants » qui prônent la rupture avec le passé.

Mais le rituel prophétique ivoirien se répète et recommence plus que jamais dans la situation actuelle du pays, avec sans doute un autre scénario et une autre distribution marqués par la scène mondiale, pour reprendre la métaphore théâtrale de l'auteur. Comme Marc Augé l'a entrevu, les prophètes pasteurs d'aujourd'hui réactivent dans le même temps la figure transfrontalière et anticipatrice d'Harris, ce citoyen libérien, catéchiste méthodiste parlant un anglais impeccable, nourri de la lecture de la Bible, et porteur des annonces d'un messianisme biblique panafricain. L'itinéraire personnel de ce contemporain de Blyden, partisan convaincu des idées d'émancipation répandues dans l'État colonial afro-américain qu'était le Libéria, illustre de façon exemplaire et, si l'on peut dire, prophétique, toutes les contradictions et les impasses du lettré africain, «chrétien et civilisé", de la première heure, militant politique nationaliste, et soudain visité, à la suite de son arrestation et de son emprisonnement, par l'ange Gabriel, élu "Prophète des temps derniers» et hanté par le retour au paganisme de ses pères.

L'ivoirisation de ce personnage charismatique légendaire a pu conduire à l'assimiler à un "prophète paysan " analphabète interdisant la lecture de la Bible-objet à ses disciples, à l'image du défunt Papa Nouveau. Mais les fils de ce dernier, n'en déplaise à l'ethnologue, ont ouvert «le Livre " et y ont trouvé la clé de la "crise spirituelle » de la nation ivoirienne. Le monde des pasteurs de guerre du «combat spirituel » qui ont porté et conseillé Gbagbo est malheureusement parfaitement "contemporain» de celui d'Harris, autant que les pasteurs évangéliques qui entourent les présidents américains. Marc Augé évoque en note, à propos de la fin du régime Gbagbo, l'«échec politique d'une génération» (p. 135). Mais l'ami Gbagbo, l'ancien résistant au "Vieux ( Houphouët), l'historien laïque et républicain, l'homme de gauche socialiste, est aussi celui qui s'est converti à la lecture quotidienne de la Bible et a été promu par ses pasteurs prophètes au rang de "premier Président chrétien de Côte-d'Ivoire", possédé par l'Esprit-Saint et surtout par les esprits de persécution des gens 
du Nord, ultime sauveur de la Nation. La contemporanéité générationnelle va décidément de pair avec l'ambivalence qui travaille les trajectoires biographiques, et la vie en double n'est jamais éloignée de la vie " en diable».

Centre national de la recherche scientifique Institut interdisciplinaire du contemporain, Paris andre-mary@wanadoo.fr

MOTS CLÉS/KEYWORDS: Marc Augé - écriture/writing - possession - terrain/fieldwork prophète/prophet. 